\title{
Self-chemisorption of azurin on functionalized oxide surfaces for the implementation of biomolecular devices
}

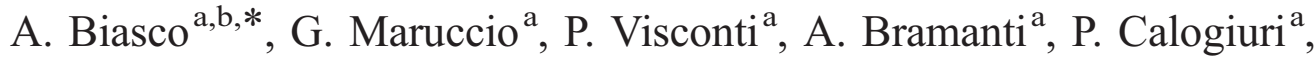 \\ R. Cingolani ${ }^{\mathrm{a}}$, R. Rinaldi ${ }^{\mathrm{a}}$ \\ ${ }^{a}$ National Nanotechnology Laboratory of INFM, University of Lecce, Via per Arnesano, 73100 Lecce, Italy \\ ${ }^{\mathrm{b}}$ ISUFI, Innovative Materials and Technology School, University of Lecce, Via per Arnesano, 73100 Lecce, Italy
}

Received 10 June 2003; accepted 16 February 2004

Available online 22 April 2004

\begin{abstract}
In this work, we investigate the formation of redox protein Azurin (Az) monolayers on functionalized oxygen exposing surfaces. These metallo-proteins mediate electron transfer in the denitrifying chain of Pseudomonas bacteria and exhibit self-assembly properties, therefore they are good candidates for bio-electronic applications. Azurin monolayers are self-assembled onto silane functionalized surfaces and characterized by atomic force microscopy (AFM). We show also that a biomolecular field effect transistor (FET) in the solid state can be implemented by interconnecting an Azurin monolayer immobilized on $\mathrm{SiO}_{2}$ with two gold nanoelectrodes. Transport experiments, carried out at room temperature and ambient pressure, show FET behavior with conduction modulated by the gate potential.

(C) 2004 Elsevier B.V. All rights reserved.
\end{abstract}

Keywords: Adsorption; Scanning microscopy; Surface roughness; Transport properties

\section{Introduction}

Biomolecular electronics is an interdisciplinary field that concerns the application of biological systems in electronic and optoelectronic devices. Application of biological systems would provide a pathway toward the miniaturization of electronic devices down to the nanometer level and toward the generation of a densely packed biomolecular nanochip [1]. In particular, metalloproteins and metalloenzymes are biological polymers that catalyse the oxidation or reduction of substrate molecules via an active redox centre containing a metal ion. Hence, the way in which electrons move through protein strands has attracted wide attention [2].

The past two decades have witnessed a growing interest in new methods of integrating solid state devices with biomolecules that present unique electronic functions. Among biomolecules, proteins have properties that make them good candidates for the assembly of nanoscale elec-

* Corresponding author. National Nanotechnology Laboratory of INFM, University of Lecce, Via per Arnesano, 73100 Lecce, Italy. Tel.: +39-832-321877; fax: +39-832-326351.

E-mail address: adriana.biasco@unile.it (A. Biasco). tronic devices. First of all, they are composed of small covalently linked monomeric subunits of relatively few kinds (amino acids) by means of which they can recognize each other, self-assembly, react specifically with surfaces or other molecules. Secondly, proteins can be further engineered to tailor their properties for nanodevices [3].

Redox proteins and enzymes are attractive targets as electron conductive materials for bioelectronics. Proteinmediated electron transfer, the essential process carried out by these molecules, has been increasingly investigated since they play a significant role not only in cellular processes but also in biotechnological applications.

In this work we face the problem of exploiting redox proteins as molecular wires that promote a strong coupling between the redox centres of two proteins or a protein and an electrode. From a practical point of view, azurin (Az) proteins have been adsorbed on the insulating gap between two metal electrodes through an organic linker in order to create a protein array (or a protein monolayer) through which electrons can flow. The aim is to fabricate a field effect transistor, working in air and at room temperature, which has redox proteins as the active layer and allows to modulate the current between the source and drain electrodes by means of the gate voltage. 


\section{Design and fabrication}

Recently, different molecular electronic devices have been fabricated that make use of individual molecules to perform electronic functions now performed by semiconductor devices [4-6]. In a previous work we demonstrated a field effect transistor based on a modified DNA base entrapped in the gap between source and drain electrodes [7].

In this perspective, the use of electron-transfer proteins appears to be particularly attractive due to their natural charge transfer activity in biological systems.

A special kind of electron transfer (ET) reaction is the reaction between the reduced and the oxidized form of the same protein, the so-called electron self-exchange (ESE) reaction.

In this work we use the blue copper protein Pseudomonas aeruginosa azurin $(14.6 \mathrm{kDa})$, one of the best characterized redox metalloproteins [8]. It mediates electron transfer in the denitrifying bacteria; specifically, it transports electrons from cytocrome $c_{551}$ to nitrite reductase in the electron transport chain of respiratory phosphorilation [9]. The central $\mathrm{Cu}$ atom, the redox active site, is coordinated to five aminoacid ligands in a distorted trigonal bipyramid geometry [9]. The tertiary structure of azurin is strongly asymmetric for the presence of a $\mathrm{S}-\mathrm{S}$ bridge opposite to copper, as clearly shown in Fig. 1. Azurin from $P$. aeruginosa fold into an eight-stranded Greek key $\beta$-barrel motif with only a $\alpha$-helix present. Given to this secondary structure and also to the disulfide bond, it can be regarded to as a very stable protein. Due to their physical and chemical properties, Az proteins are good candidates for bioelectronic applications.

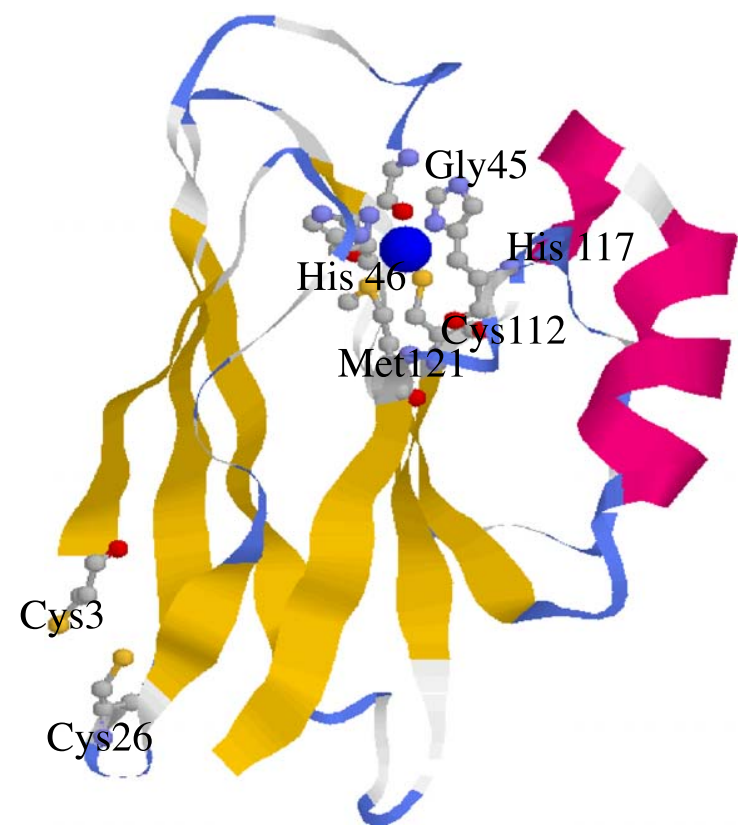

Fig. 1. 3D structure of $P$. aeruginosa azurin. Opposite to $\mathrm{Cu}$ atom there is the unique disulfide bridge between Cys3 and Cys26. Alpha helices are evident on the right. Five aminoacids coordinate the $\mathrm{Cu}$ ion giving a distorted trigonal bipyramid geometry.

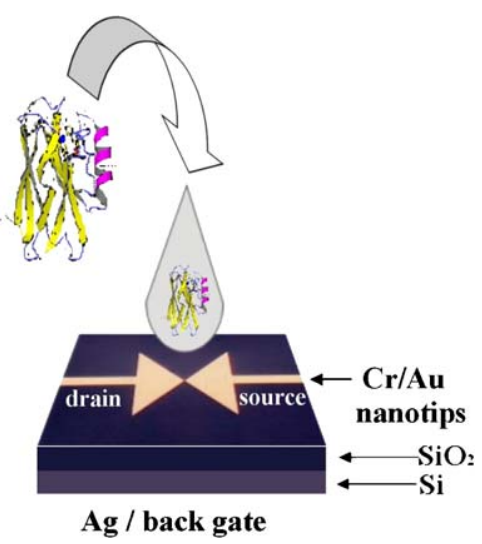

Fig. 2. Azurin protein solution is deposited onto the gap.

The immobilization procedure is a particularly demanding aspect: it should assure an appropriate and permanent localization of biomolecules on a support and it must preserve its necessary function [10]. Different immobilization methods can be employed in order to attach the protein to the substrate: among others (physical entrapment within membranes, Langmuir Blodgett, physical or chemical adsorption), covalent attachment on self assembled monolayers (SAMs), consisting of molecules adsorbed from solution onto solid substrates [11], are the most promising since they provide control over the reproducibility and orientation of the anchored protein.

Apart from being oriented and higly ordered, SAMs are chemically and thermodynamically stable even after coupling them with the immobilising molecule. They can also be prepared in laboratory using very simple method and equipment.

By immobilizing proteins in thin monolayer films of polymers, it is possible to interface molecules with metallic electrodes. Chemical functionalization of oxygen-exposing surfaces by means of linker molecules creates bio-compatible interfaces that communicate with metallic circuits.

Three-terminal nanodevices were fabricated using a combination of photolithography and electron-beam lithography (EBL), as previously described [12]. They consist of two $\mathrm{Cr} / \mathrm{Au}(8 \mathrm{~nm} / 35 \mathrm{~nm})$ tip-shaped electrodes on a $\mathrm{Si} / \mathrm{SiO}_{2}$ substrate with a tip separation between 100 and $10 \mathrm{~nm}$.

The technique for protein immobilization is based upon the self-assembly of 3-MPTS on $\mathrm{SiO}_{2}$ surfaces. A very diluted solution $(2 \%)$ of 3 -MPTS has been used to obtain ordered monolayers and to prevent from the multilayer formation. Sulfide groups are adsorbed from high purity ethanol solution and at the solid-liquid interface a strong chemisorption of the head group (Si) gives rise to highly ordered architectures.

The SAMs were constructed by using a two-step procedure. Substrates were incubated in 3-MPTS ethanol solution. The promoted surfaces were exposed to commercial $\mathrm{Az}$ solutions and gently dried at room temperature. Adsorption of $\mathrm{Az}$ was performed from $10^{-4} \mathrm{M}$ solution 
with a $\mathrm{pH} 4.6$ prepared dissolving $\mathrm{Az}$ in $50 \mathrm{mM}$ ammonium acetate buffer. The choice of a $4.6 \mathrm{pH}$ value is due to the fact that it corresponds to the Az isoelectric point. In these conditions no charges are present on the biomolecule surface and the adsorption process is not affected by electrostatic attractive or repulsive forces. Fig. 2 shows the deposition of the azurin solution on the final device and between the electrodes.

\section{Characterization and discussion}

In the present work we took advantage of atomic force microscopy (AFM) to provide qualitative information about adsorbed proteins with molecular resolution [13].

The SAM preparation was performed on the cleaned and dried surfaces (pre-treated with a oxygen plasma treatment) in absolute ethanol solvent and in a clean and dust-proof environment.

3-MPTS was used as a cross-linking agent because it ensures orientation of the protein deposited film. In fact, the protein adsorption on 3-MPTS SAM occurs by the unique disulfide bridge. Therefore, proteins are well ordered in the nanomolecular film and the molecular architecture is similar to that of immobilized protein molecules on gold [14].

Fig. 3 displays the obtained tapping-mode AFM images with a scan size of $0.88 \times 0.88 \mu \mathrm{m}$.

This is an evidence for azurin adsorption since it reveals features uniformly distributed across the surface, which were not present on SAMs substrates (images not shown). The presence of these topographic features was reflected in the roughness. The protein adsorption, in fact, is responsible for a surface root mean square (rms) roughness increasing from 0.3 to $3.17 \mathrm{~nm}$. The recognizable granular structures are adsorbed proteins and the surface is totally covered by proteins. The images have not been processed with the exception of flattening.

AFM techniques can add size and shape to imaged structures due to tip convolution effect; consequently, the lateral size of nanoparticles dispersed onto a substrate results overestimated. The real surface topography could be obtained only by using an infinitely sharp tip (zero curvature radius) or, as in the specific case of adsorbed proteins, by performing AFM imaging in a liquid cell containing physiological buffer [15]. In our experiments, since the tip could not be reduced under a certain limit and since a dried environment was requested, we used an approximation to find out protein real dimensions. It is known that $\mathrm{Az}$ has a dimension of $4.4 \mathrm{~nm}$ [14]. If we approximate the tip to a sphere of diameter $D$ and the protein to a sphere of actual diameter $d$, then the apparent width $W$ can be calculated from the formula [16]

$W=2(D d)^{1 / 2}$.

The nominal $D$ value is $20 \mathrm{~nm}$, therefore the apparent width measured by AFM (19-21 nm) corresponds to $d=4.5-5.5 \mathrm{~nm}$, not very different from the literature value. On the contrary, the height measured is not affected by the size of the AFM probe. The average height is $3.26 \mathrm{~nm}$ that is lower then the expected one. This could be explained by taking into consideration the compression over the protein
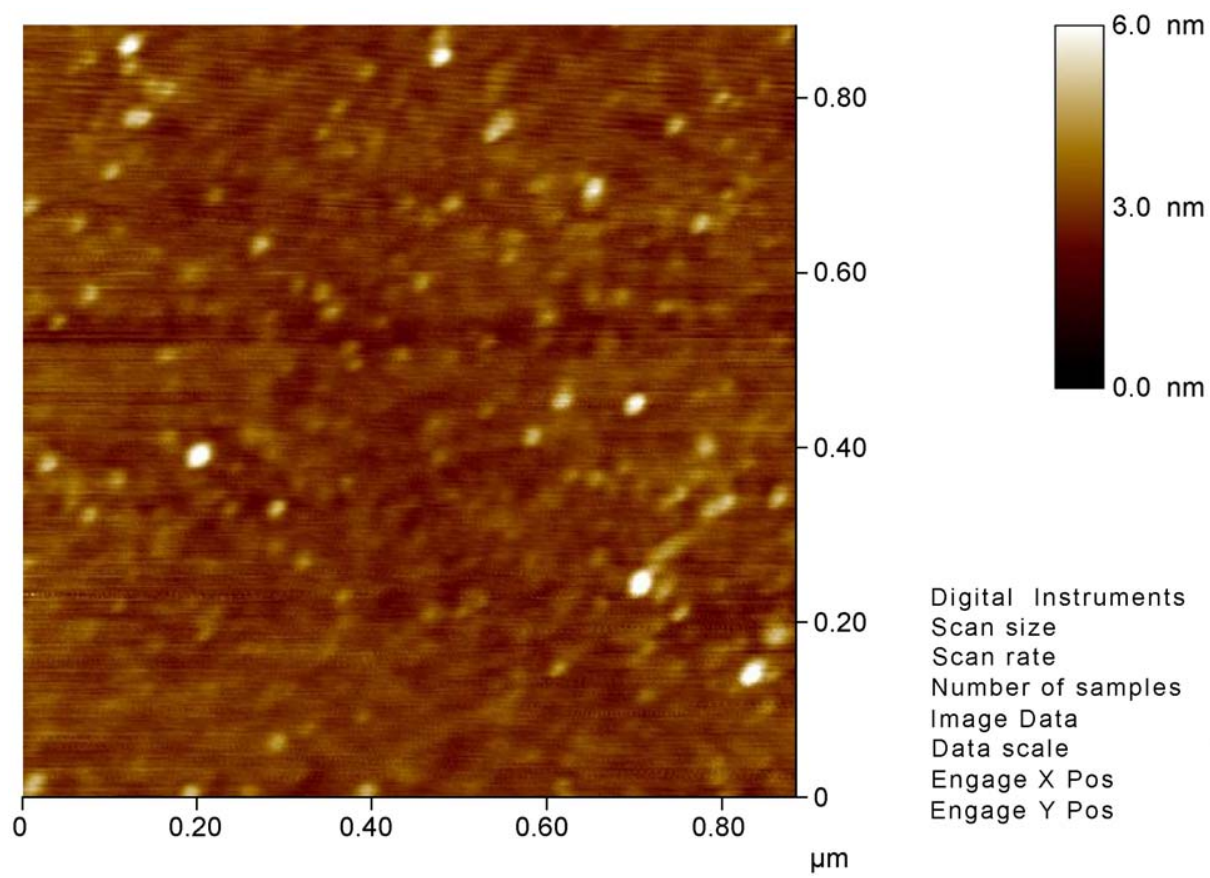

$\begin{array}{lc}\text { Digital Instruments } & \text { Nanoscope } \\ \text { Scan size } & 0.8848 \mu \mathrm{m} \\ \text { Scan rate } & 0.9988 \mathrm{~Hz} \\ \text { Number of samples } & 512 \\ \text { Image Data } & \text { Height } \\ \text { Data scale } & 6.000 \mathrm{~nm} \\ \text { Engage X Pos } & \\ \text { Engage Y Pos } & \end{array}$

Fig. 3. About $0.88 \times 0.88 \mathrm{~mm}^{2}$ topography image of a protein adsorbed surface. It was recorded using a Bio Scope AFM (Veeco Metrology Group) in the noncontact mode. The scan rate was $1 \mathrm{~Hz}$ and the set point $2.9 \mathrm{mV}$; (columns $\times$ rows $=512 \times 512$ ). 
induced by tip force while scanning the surface and the distortion effect after the adsorption and drying process.

Current-voltage experiments were carried out by using a semiconductor parameter analyzer (HP Agilent 4155B) in the voltage range between -6 and $6 \mathrm{~V}$, at room temperature and ambient pressure. The gate voltage $\left(V_{\mathrm{G}}\right)$ was changed in order to investigate its influence on the current $\left(I_{\mathrm{ds}}\right)$ between the source (s) and drain (d) electrodes. Before the deposition of molecules, all devices were tested to verify that the channel was insulated (resistance as large as $100 \mathrm{G} \Omega$ ).

Among all the investigated samples, only few behaved as a FET since a modulation of the transport between $S$ and $D$ voltage by means of the gate electrode was observed. In most cases, in fact, protein solution is physically excluded from the few nanometer gap between electrodes and this prevents from obtaining an azurin active layer. A typical current-voltage $(I-V)$ curves measured in air at room temperature is shown in Fig. 4. The figures represent the $I_{\mathrm{ds}}$ current vs. $V_{\mathrm{ds}}$ voltage at different $V_{\mathrm{g}}$ voltages. $V_{\mathrm{ds}}$ was varied between 0 and 6 in one case (a) and between 0 and -6 (b) in the other. In both cases a low-current plateau is discernible meaning that our system behaves like an insu- lator at low voltages. Then current rises steeply above a threshold voltage of the order of $3 \mathrm{~V}$ and saturates. At a fixed value of $V_{\mathrm{ds}}=4.5 \mathrm{~V}$ it has been observed that $I_{\mathrm{ds}}$ increases with $V_{\mathrm{g}}$, it reaches a maximum at approximately $V_{\mathrm{g}}=1.2 \mathrm{~V}$ and then decreases. This behaviour is not evident in semiconductor devices and is certainly due to the presence of the biological material. Moreover, it could be exploited for the fabrication of nanodevices with new functionalities, unknown to the semiconductor industry.

Azurin monolayers chemisorbed on functionalized oxide surfaces have been fabricated successfully and assessed by AFM. A blue-copper protein modified field effect transistor is presented. The characteristic of the developed device is described. We suggest that transport through the protein copper site is responsible for conduction whereas the modulation is correlated to the field effect. However, after some cycles of measurement, current level drops because of device aging, due to both molecular active layer degradation and electrode burning. As revealed by SEM investigations (images not shown), a correlation between electrical response and morphological changes of the molecular active layer is observed. Though a better control of the main parameters affecting the reproducibility has to be achieved,
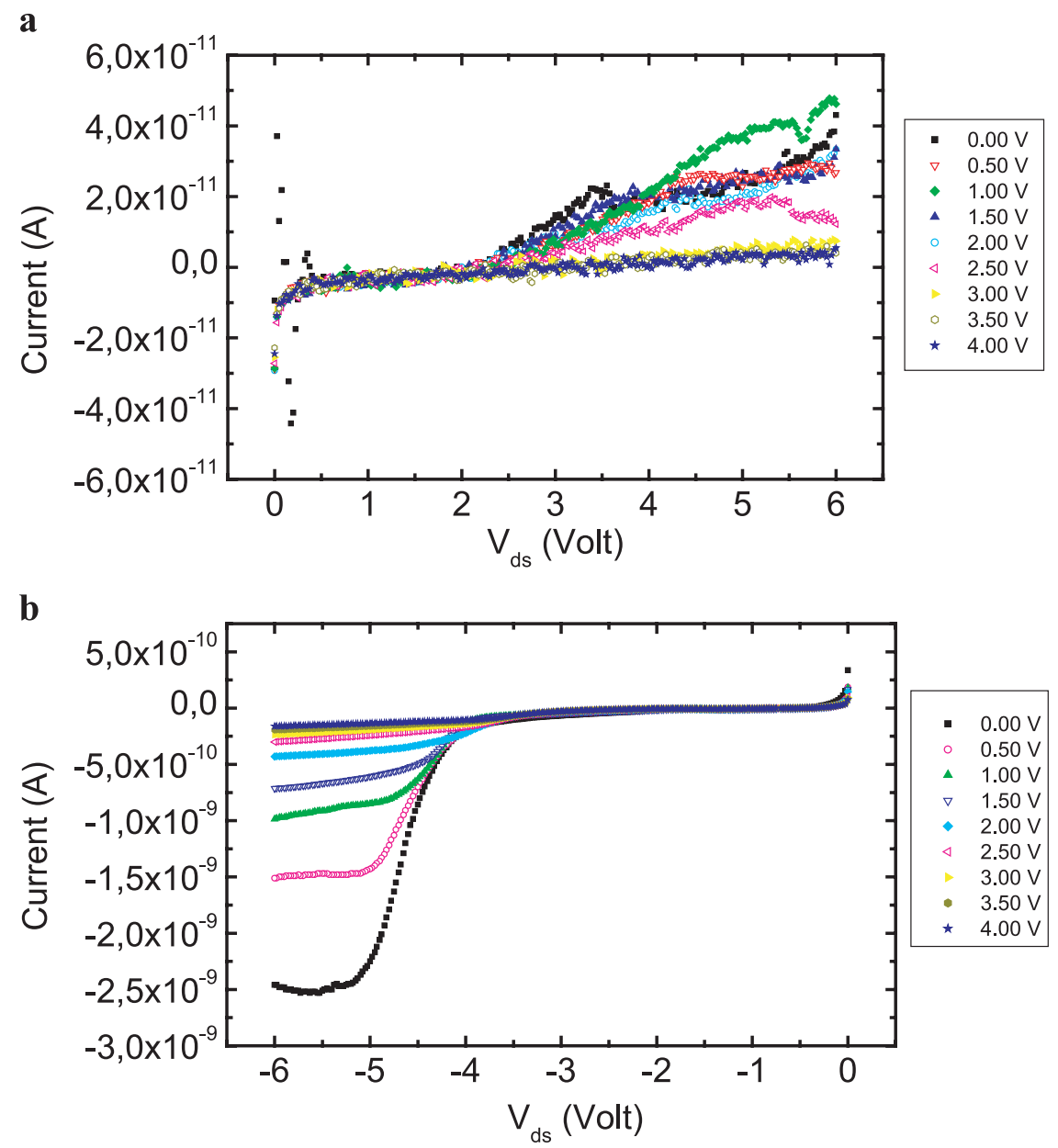

Fig. 4. (a) $\mathrm{I}-\mathrm{V}$ characteristics of an azurin device. $V_{\mathrm{ds}}>0, V_{\mathrm{g}}>0$. (b) $\mathrm{I}-\mathrm{V}$ characteristics of an azurin device. $V_{\mathrm{ds}}<0, V_{\mathrm{g}}>0$. 
the demonstration of such field effect device is an important step towards the development of molecular electronics.

\section{Acknowledgements}

This work is the result of recent research carried out by the biomolecular electronics division of the National Nanotechnology Laboratory (NNL)-Lecce. Special thanks go to the team of the European Community project SAMBA. Financial support by EC through SAMBA project is gratefully acknowledged.

\section{References}

[1] A.B. Kharitonov, M. Zayats, A. Lichtenstein, E. Katz, I. Willner, Enzyme monolayer-functionalized field-effect transistors for biosensor applications, Sens. Actuators, B, Chem. 70 (1-3) (2000) 222-231.

[2] M.L. Kennedy, B.R. Gibney, Metalloproteins and redox protein design, Curr. Opin. Struck. Biol. 11 (4) (2001) 485-490.

[3] G. Gilardi, A. Fantuzzi, Manipulating redox systems: application to nanotechnology, Trends Biotechnol. 19 (11) (2001) 468-476.

[4] C. Joachim, J.K. Gimzewski, A. Aviram, Electronics using hybridmolecular and mono-molecular devices, Nature 408 (2000) 541-548.

[5] J.M. Seminario, A.G. Zacarias, J.M. Tour, Theoretical study of a molecular resonant tunneling diode, J. Am. Chem. Soc. 122 (13) (2000) 3015-3020.

[6] M.A. Reed, J. Chen, A.M. Rawlett, D.W. Price, J.M. Tour, Mo- lecular random access memory cell, Appl. Phys. Lett. 78 (23) (2001) $3735-3737$.

[7] G. Maruccio, P. Visconti, V. Arima, S. D’Amico, A. Biasco, E. D'Amone, R. Cingolani, R. Rinaldi, S. Masiero, T. Giorni, G. Gottarelli, Field effect transistor based on a modified DNA base, Nano Lett. 3 (4) (2003) 479-483.

[8] E.T. Adman, R.E. Stenkamp, L.C. Sieker, L.H. Jensen, A crystallographic model for azurin at $3 \AA$ resolution, J. Mol. Biol. (123) (1978) $35-47$.

[9] M.A. Webb, C.M. Kwong, G.R. Loppnow, Excited-state charge-transfer dynamics of Azurin, a blue copper protein, from resonance raman intensities, J. Phys. Chem., B 101 (25) (1997) 5062-5069.

[10] N.K. Chaki, K. Vijayamohonan, Self-assembled monolayers as a tunable platform for biosensor applications, Biosens. Bioelectron. $17(1-2)(2002) 1-12$.

[11] C.H. Kobler, D.K. Scwartz, Langmuir and self-assembled monolayers, Curr. Opin. Colloid Interface Sci. 4 (1) (1999) 46-51.

[12] G. Maruccio, P. Visconti, S. D’Amico, P. Calogiuri, E. D’Amone, R. Cingolani, R. Rinaldi, Planar nanotips as probes for transport experiments in molecules, Microelectron. Eng. 67-68 (2003) 838-844.

[13] J.J. Davis, H.A.O. Hill, The scanning probe microscopy of metalloproteins and metalloenzymes, Chem. Commun. (2002) 393-401.

[14] B. Schnyder, R. Kotz, D. Alliata, P. Facci, Comparison of the selfchemisorption of azurin on gold and on functionalized oxide surfaces, Surf. Interface Anal. 34 (1) (2002) 40-44.

[15] P. Facci, D. Alliata, L. Andolfi, B. Schnyder, R. Kotz, Formation and characterization of protein monolayers on oxygen-exposing surfaces by multiple-step self-chemisorption, Surf. Sci. 504 (2002) $282-292$

[16] J. Fang, C.M. Knobler, M. Gingery, F.A. Eiserling, Imaging bacteriophage T4 on patterned organosilane monolayers by scanning force microscopy, J. Phys. Chem., B 101 (43) (1997) 8692-8695. 